\title{
MOBILE LASER SCANNER MAPPING SYSTEM'S FOR THE EFFICIENCY OF THE SURVEY AND REPRESENTATION PROCESSES
}

\author{
A. Dell'Amico' ${ }^{1}$ \\ ${ }^{1}$ DICAr, The Department of Civil Engineering and Architecture, University of Pavia, Italy - anna.dellamico@unipv.it
}

KEYWORDS: Mobile Laser Scanner, Fast Survey, Cultural Heritage, Drawing, KAARTA, 3D Database.

\begin{abstract}
:
The research activity aims to evaluate the Mobile Laser Scanner mapping system's effectiveness and critical issues based on simultaneous localization and mapping (SLAM) called KAARTA Stencil. The research introduces a reflection on a series of test datasets resulting from the mobile system's application in an urban context taken as a case study and aimed at the representation and 3D modeling of architectural complexes.

In detail, the metric accuracy of the proposed method was evaluated through a comparative analysis of the point cloud data through the evaluation of the surface deviation of the 3D point clouds based on the SLAM system and the data of static measurement systems, more precise to evaluate the accuracy of the proposed acquisition system. For each data were specified the possibilities of representation, the type of representation scale, and the possible manipulations and extractions of 2D profiles to design and analyze architectural elements through mobile systems.

The analysis involves a breakdown of the problem of representation, identifying protocols that can be applicable at different levels and scales of reading aimed at the representation and discretization of parts and elements linked together in a hierarchical or interconnected relationship, outlining their criticality and potential.
\end{abstract}

1.

\section{INTRODUCTION}

Research on the representation of architectural heritage through the construction of digital databases for is broadening its horizons and developing innovative tools and techniques for rapid acquisition. In support of the attempt to reduce the times for the extraction of two-dimensional and three-dimensional data by quickly providing data that can be used to develop BIM procedures. BIM modelers use point clouds to extract the physical and functional information of the case studies of interest. The multiple points of view obtained from the point cloud scan data provide holistic visibility of the structure, guaranteeing the accuracy of the representation.

Specifically, the request for information annexation creates a reflection between scientific and methodological issues ranging from the choice of data acquisition tools to their use and representation techniques.

The study of methodologies and tools "must also pay attention to the specific cognitive problems about architectural representation to take into account the connection between perception and the semiotic foundations of communication" (De Luca et al., 2014).

The metric acquisition phase of cultural heritage using rangebased techniques such as terrestrial and mobile laser scanners and Structure from Motion photogrammetric techniques, referring to range based models remains the initial phase for the construction of the primary data for reconstructive digital modeling activities aimed at heritage conservation historicalarchitectural. Although data collection techniques are now very efficient and are increasingly directed towards process automation, digital representation methods through critical data processing pipelines require, despite the development of advanced software, a long time to process digital models through modeling processes and reverse engineering techniques (Lo Turco et al., 2021).
Through this research survey, a reflection is posed by the relationship between the display systems for representation within the same graphic space and the morphological complexity and quality of the information readable by the data of the mobile laser scanner.

The decoding of spatial continuity, while drawing, will never be perfectly adherent to the real form but will be the result of a summation of systematic errors. The data undergoes a first translation and deformation during the instrumental acquisition phase in which the first translation takes place (instrumental errors). Here it happens a first phase of discretization based on two limits: the first is of the instrumental type represented by the number of points that the instrument can acquire, the second is of the subjective type represented from the acquisition choices made by the detector as well as the observer.

The transition from the continuity of reality through digital acquisition takes the form of a database consisting of points and spatial coordinates, which are optimized to achieve good uniformity of data quality. The digital space, ideally unlimited, assumes a limitation given by the hierarchical composition of the elements that compose it. An additive type composition can be implemented with other forms of data generated by the different methodological applications used to understand the architectural elements.

The need for a digital representation arises from a will, a specific request, the two things cannot be released. The need for a specific type of analysis is the driving force behind the relevant activities, which are consequently designed on the basis of specific objectives.

On the basis of this trend, a reflection is placed on the relationship between instrumental innovation and systems of representation. The different instrumental possibilities available in the cultural heritage sector can constitute new tools for scientific investigation and visualization, taking into account the dimensions of reading the complexity and intelligibility within 
the graphic representation space. Modern information databases use digital survey techniques to realize the knowledge base and the qualification and quantification of the spaces translated into the spatial coordinates of the point clouds. This 3D data is typically used for accurate documentation, digital preservation, visualization and analysis.

The evaluation of the type of mass capture device must be carried out based on the geometries of the architectural object elements, which must be legible within the metadata of the point cloud (López et al., 2020). The most influential instrumental characteristics are resolution, accuracy, timing, and the availability of the RGB texture of the points.

Nowadays, the amount of 3D datasets available has increased exponentially by tools divided about the character of the survey operations from the macro-scale level to the micro-scale survey level.

The tools and procedures year after year refine sensors and techniques trying to make the processes from the acquisition of raw data to the reprocessing of these as automated as possible. The aim is to define low-cost procedures based on the concepts of traditional survey extended to the instrumental acquisition, reducing timing and offering the end-user a product "easy" to use, and guaranteeing a reliable result from a metric point of view (Apollonio et al., 2010).

\section{METHODOLOGY}

The trial aims to evaluate the effectiveness and critical issues of the Mobile Laser Scanner mapping system based on simultaneous localization and mapping (SLAM) KAARTA Stencil. The study saw the implementation of application tests aimed at verifying the product in qualitative and quantitative terms, taking into account the methods, the timing of recovery, and the quality of the data corresponding to the different types of acquisition.

Defining a data acquisition methodology that can be applied, through the validation of the acquisition processes, aimed at the extensive survey on a large urban and territorial scale, evaluating the potential and the planimetric accuracy and elevation of the mobile data for the use of these types of systems in the field of city modeling representation and urban planning (Toschi et al., 2017).

In recent years, mobile 3D mapping has developed different portable solutions for close-range mapping systems, developing systems and product categories designed for rapid detection procedures and associated with scan-to-modeling operation. These systems find success in the construction sector in the various fields of application from the architectural survey, plant engineering, analysis of the territory, and management of cultural heritage (Tucci et al. 2017).

The research proposes a reflection on a series of test datasets resulting from the application of the mobile system in an urban context taken as a case study and aimed at the representation and 3D modeling of architectural complexes.

The experimentation divided by a set of analysis and data collection was aimed at analyzing the accuracy and type of information content that can be read on the point clouds of the KAARTA system, imagining the Mobile Terrestrial LiDAR System (MTLS) as possible autonomous detection solution or as a combined action through data integration by combining mobile technology with multisensor detection systems. (Dell'Amico, 2020).

In detail, the metric accuracy of the proposed method was evaluated through a comparative analysis of the point cloud data through the evaluation of the surface deviation of the $3 \mathrm{D}$ point clouds based on the SLAM system and the data of static measurement systems more precise to evaluate the accuracy of the proposed acquisition system (Tu et al., 2017). For each data are specified the possibilities of representation, the type of representation scale, and the possible manipulations and extractions of $2 \mathrm{D}$ profiles for the design and analysis of architectural elements through mobile systems (Sammartano, Spanò, 2018).

MTLS systems are emerging technologies that combine in a single instrument a laser scanner, an Inertial Navigation System (INS), and an odometry system, assembled on a mobile platform, designed for the on-the-go acquisition of geospatial data.

SLAM systems are considered, in the topographical field, to be inaccurate acquisition systems with low-quality performances. In recent years, SLAM technology has been considered within scan-model workflows to reduce production times in the representation and digitization of space, thanks to the potential to guarantee coverage of acquisition of large areas in a short time (Parrinello et al., 2019). Companies such as Leica, FARO, GeoSlam, Paracosm, NavVis, KAARTA, have launched the mobile alternative to qualitatively more high-performance TLS products on the market, given the demand for rapid acquisition systems.

These systems have an acquisition capacity of about 400000 points/sec with a range of about $25 / 30$ up to $80 \mathrm{~m}$ and different shooting angles of $360^{\circ}$ horiz. $270^{\circ}$ vertical (BLKgo, ZEB), $360^{\circ}$ horiz. $30^{\circ}$ vertical (KAARTA).

The algorithm and applications developed for mobile systems tend towards the automation of the acquisition process and the data recording process. The trend is reflected in the manufacturer's choice to include some aspects of SLAM systems that have also been included in static laser scanner systems such as in $\mathrm{Z}+\mathrm{F}$, FARO, and Leica, in which accelerometers and compasses are present. Inside the RTC 360 model manufactured by Leica is the complete visual system between scans used to help the rough alignment between contiguous scans.

Types of acquisition correspond to acquisition projects point clouds, the accuracy and level of detail required strictly depend on the objectives and representative needs specified by the project's aims.

The acquisition can provide different results depending on the movement, the shooting angle, the acquisition mode, the factors related to the environmental context, the shooting times.

If these factors are not kept under control through a context analysis and planning of the acquisitions, the data recorded through some errors of different types:

- Partial acquisition of the data.

- Deviation of the data concerning the trajectory performed.

- Misalignment of the data.

- Duplication of the data in the same coordinate space.

These errors are mainly due to the acquisition methods during the operational fieldwork.

The device analyzed is a KAARTA Stencil type mobile laser scanner, made available by the laboratories or DAda-LAB of the Department of Civil Engineering and Architecture of the University of Pavia.

It is essential to underline that although the SLAM system is often defined as a technology, it is more appropriate to refer to this system as a methodology. The success of the processing depends on a series of factors that the operator must know before approaching this system.

The instrument uses proprietary technology, similar to SLAM technology but which aspires to have a high performance in terms of accuracy, through the integration and implementation of a 3D LiDAR sensor, IMU (inertial platform), and odometric chamber, mounted on static support that an operator can maneuver, and is equipped with a simultaneous data control monitor that allows the operator to check the shooting maneuvers constantly. It manages to acquire in a range of about 80 meters about 400,000 points per second, it should be emphasized that the field of view of the instrumental shooting 
angle is limited it imposes an angular shooting limit $\left(360^{\circ}\right.$ horizontal FOV $30^{\circ}$ (from $+15^{\circ}$ to $-15^{\circ}$ ) vertical FOV) for which it is necessary to structure the types of path weighted on the acquisition object. The quality of the data of the MTLS point clouds is strictly dependent on the way the operator maneuvers the instrument during the acquisition phase, on the times and paths chosen for the recovery. To ensure the success of the shooting activities, the operator must know how to maneuver the instrument with awareness and caution by tilting the instrument's head in a weighted manner according to the acquisition objective.
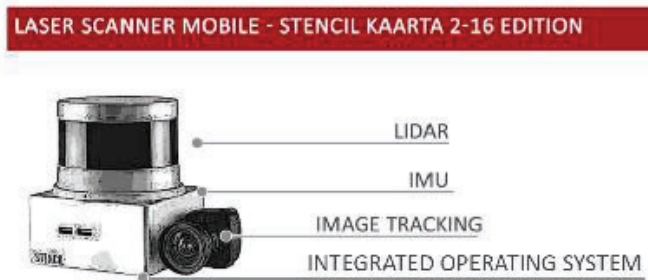

Technical specifications
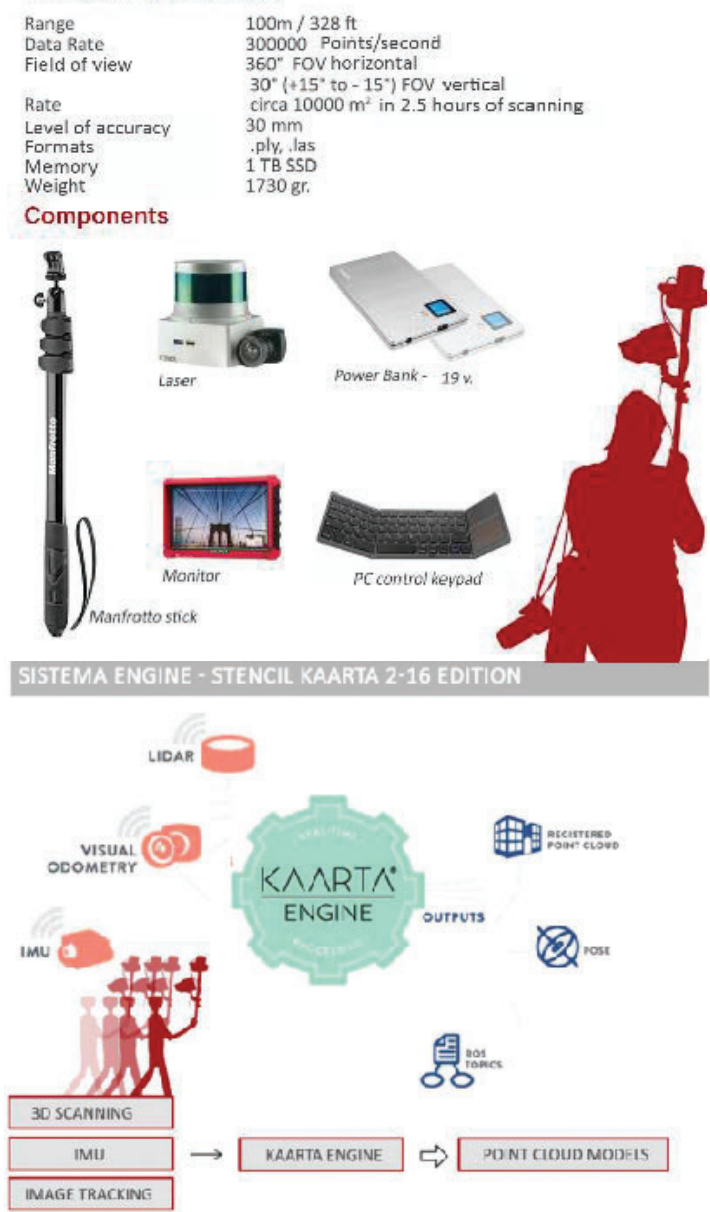

Figure 1. The KAARTA mobile laser scanner system. The instruments is based on proprietary SLAM technology that works through the superimposition of data, i.e. on simultaneous localization and mapping and at the same time allow you to estimate the position of the instrument and generate a digital model of the detected scene.

For this reason, the trajectory and the acquisition loops must be planned according to the local environment configuration. This action is fundamental because the SLAM-based system exploits the iterative alignment of the extracted profiles obtained on those characteristic attributes of the space. The acquisition time is considered the sum of the few minutes necessary for initialization and closing with pre-processing and automatic storage on site of the data in the embedded memory and the execution of the trajectory. Considering an operator walking on foot with a handheld device with a speed of nearly $1-1.5 \mathrm{~m} / \mathrm{s}$, a route of up to 20-30 minutes is recommended to ensure better accuracy of the result data.

Therefore, the structuring of the paths aims to guarantee coverage on the horizontal and vertical plane of the data of the point cloud and provide for a good refinement of the data to increase the reading quality (Parrinello et al., 2020).

Given the instrumental limit of vertical shooting of $30^{\circ}$, it is necessary to repeat the combined path trajectory and the instrumental angle of inclination. The scan output of the single shooting slot will actually be the combined result of the sum of different shooting inclinations recorded in an automated manner by the instrumental algorithm.

\subsection{Case study}

The street front examined for the methodological experimentation comprises nine building units in line, which are an excellent example of the complexity and heterogeneity of the buildings on the street front.

For the analysis of the data and the processes of representation, starting from the cloud of points, a portion identified by a building unit was chosen, which includes the coexistence of different masonry textures on the wall front in addition to the presence of urban furniture elements, signs, electrical systems, gutters, and downspouts. The front is spread over three levels: the base is made of "facing" stone with blocks of various sizes interrupted by access portals to commercial funds and service doors of the residential floors, the two elevation floors have a material treatment in plastered brick and have six regular openings, three for each level.

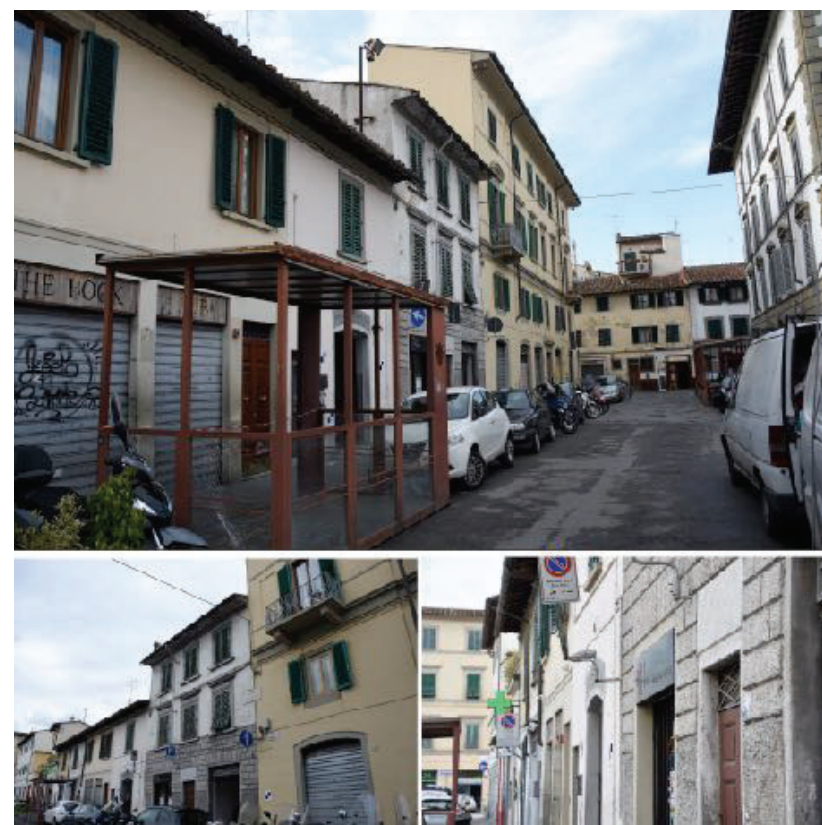

Figure 2. Some framing photos of the case study chosen for the experimentation, in particular it is via mino, historic center of

Florence, Italy.

On the first floor single-lancet windows with entablature and on the second floor with molded frame, in which there is no architrave. To divide it from the carriageway, a pedestrian sidewalk, and an area reserved for parking cars and motorcycles. The representative scheme of the road-building system and pedestrian and vehicle area has influenced the design of the routes by conveying and forcing the choice of 
trajectories along the lane of the main carriageway and the pedestrian sidewalk.

\subsection{Acquisition methodology}

As a preliminary to the acquisition phases, five black \& white targets were positioned on the front to recognize homologous points useful for checking significant points in the subsequent data recording and analysis phase.
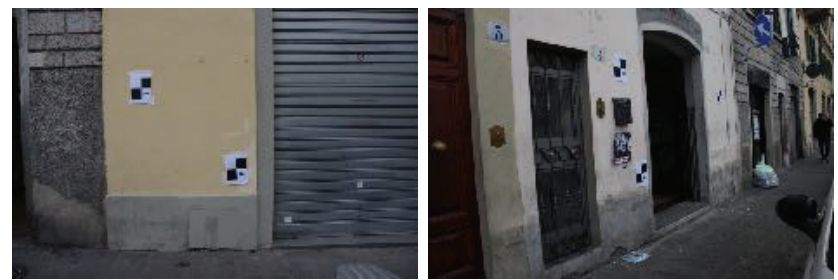

Figure 3. Positioning of Black \&White targets for the recognition of detection points.

The operations of acquiring the street front with the mobile laser scanner were designed to carry out a comparative analysis of different types of output resulting from methodically increasing the trajectory paths and shooting times.

Four shooting slots have been provided. The trajectory paths have been increased exponentially, providing for an instrumental inclination change of about $30{ }^{\circ}$ for each trajectory, keeping the instrument tilted in the same position:

- The first slot (K1) provided a single trajectory in the driveway center, keeping the instrument in a vertical position of about $90^{\circ}$ at the height of about $1.90 \mathrm{~m}$ from the ground, for a total of 2 minutes of shooting, producing a cloud of points ( 10033773 p.) In terms of data readability, which covered the road front up to the first level. to evaluate the instrumental visual coverage range.

- The second slot (K2) provided two trajectories along the driveway edges, keeping the instrument in a vertical position of about $90^{\circ}$ at the height of about $1.90 \mathrm{~m}$ from the ground, for a total of 3 minutes of shooting, producing a cloud of points $(16942452$ p. ). The slot increased data covered by the passage of the first trajectory by about $10 \%$, covering the first level and partially the second in terms of readability. But still presenting an evident lack of data in the portions over 3 meters high due to a lack of variation of the instrumental visual angle.

- The third slot (K3) provided for four trajectories repeating the paths of the second shooting slot; two by keeping the instrument vertical at $90^{\circ}$ and two by varying the instrumental shooting angle by about $30^{\circ}$ degrees $\left(90^{\circ}+30\right.$ ${ }^{\circ}$ ); for a total of 6 minutes of shooting. The generated point cloud counts 34252323 points do not show any misalignments due to changes in trajectory and inclination and shows readability increased by $20 \%$ compared to the previous two. The eaves line is visible in this case, but the third level is still poorly defined and legible in detail.

- The fourth slot (K4) provided eight trajectories; the four trajectories of the third slot were repeated, and to these were added two trajectories along the sidewalk adjacent to the street front and two trajectories along the opposite sidewalk. In this case, every two trajectories the instrumental inclination angle was changed, increasing the shooting angle by about 30 $\circ$ for each trajectory change while maintaining the height of the instrument constant at about $1.90 \mathrm{~m}$ from the ground. The operation involved a total of 9 minutes of shooting, generating a cloud that counts 67479745 points. In this case, the data covers the entire wall front; the third level increases the more defined quality of detail by $20 \%$ compared to the previous acquisition. Also, in this case, the definition line of the eaves level is visible, except for the roof pitch, which is an undetectable portion following the chosen path trajectories. In the end the mobile data were compared with a TLS acquisition point cloud acquired using a FARO Cam Focus S150 laser scanner. The global registration counts 11 scans, done along the street front by setting an RGB recovery of the data, and each scan provided an acquisition time of $6 \mathrm{~min}$. for a total time of about one hour and six minutes. The data is of higher quality than the mobile data, also enriched by the colorimetric character. At the global representation level, the data of the fourth slot is in every way competitive in terms of representation and identification of the elements, valid for the rapid representation of the fronts with times seven times less acquisition than the TLS system. The clouds were acquired with the aim of comparing shooting methods and data obtained, in order to be able to proceed with a weighted assessment and conscious standardization of the shooting processes through the use of the mobile tool.

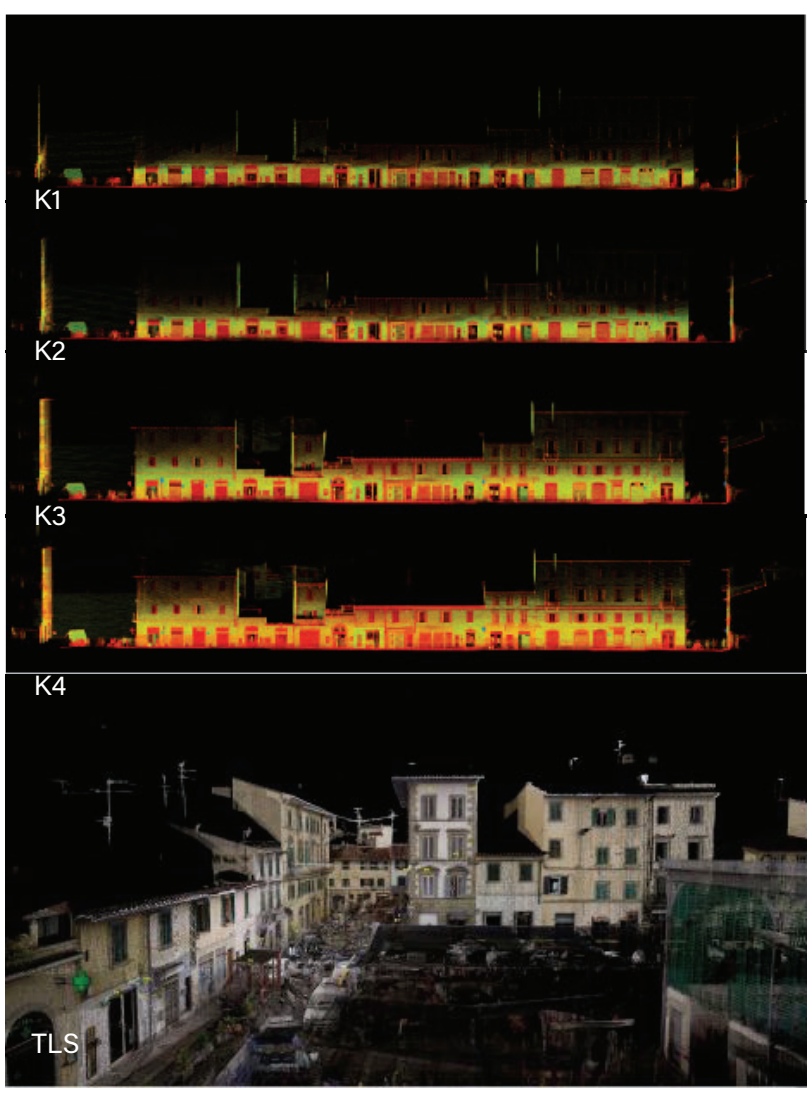

Figure 4. The operations of acquiring the street front with the mobile laser scanner were designed to be able to carry out a comparative analysis of different types of output resulting from methodically increasing the trajectory paths and shooting times. Four shooting slots have been provided, in which the trajectory paths have been increased exponentially, providing for an instrumental inclination change for each walk. 


\begin{tabular}{|l|c|c|c|}
\hline \multicolumn{1}{|c|}{ Scan name } & $\begin{array}{c}\mathrm{n}^{\circ} \\
\text { trajectories }\end{array}$ & $\begin{array}{c}\text { Acquisition } \\
\text { time }\end{array}$ & $\begin{array}{c}\mathrm{n}^{\circ} \\
\text { point }\end{array}$ \\
\hline $\mathrm{K} 1$ & 1 & $2 \mathrm{~min}$ & 10033773 \\
$\mathrm{~K} 2$ & 2 & $3 \mathrm{~min}$ & 16942452 \\
$\mathrm{~K} 3$ & 4 & $6 \mathrm{~min}$ & 34252323 \\
$\mathrm{~K} 4$ & 8 & $9 \mathrm{~min}$ & 67479745 \\
\hline
\end{tabular}

Table 1. the table shows the data regarding the single scan slots: number of trajectories, acquisition time and number of points of the generated cloud.

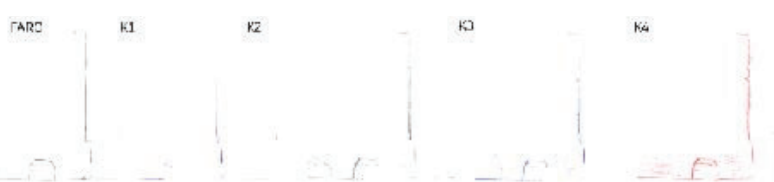

Figure 5. Section line comparison between different scan slots.

\section{POINT CLOUD DATA ANALYSIS}

In order to proceed with the evaluation of the acquired data, in terms of readability and the possibility of redrawing, the scans acquired once downloaded from the instrument and were converted from the proprietary format .ply, into .e57 format using the Cloud Compare software. The scans have been subsequently imported into the Leica Cyclone software for recording and extrapolation of orthoimages to analyze the data reading quality. Once the project was structured, the 4 scan slots were registered with each other by identifying homologous points identified by the black \& white targets. Having been strategically positioned within the first level, for visibility reasons visible in all scan slots. The point cloud acquired with the mobile tool takes the characteristic spacing of the surface points making the data with a non-millimeter accuracy index. This in the moment of recording the data through the identification of morphological vertices and the central point of the black and white target can cause alignment errors that can be found in a different readability of a point between different scans. In this case, the vertices considered to be clearly visible in the different scanning slots were evaluated to unify the different slots in a common coordinate system to be able to metrically contrast the different slots and set UCS reference system and cutting planes.

Observing the images makes it possible to see how the data is less dense and, therefore, less readable with faster scan slots. From the first scan slot to the fourth, the information is significantly increased by $40 \%$, thanks to the structuring of trajectories to improve the instrumental coverage radius and cover the occlusion points generated by the obstructions present in the stretch of road.

On a dimensional level, no distortions are noted from the metric point of view between the different shooting slots. The second action was to understand the data. A reference plane common to all slots was set. A slice and an orthoimage of the same portion were extrapolated for each scan lot to understand the relationship between scanning speed and data reading quality.
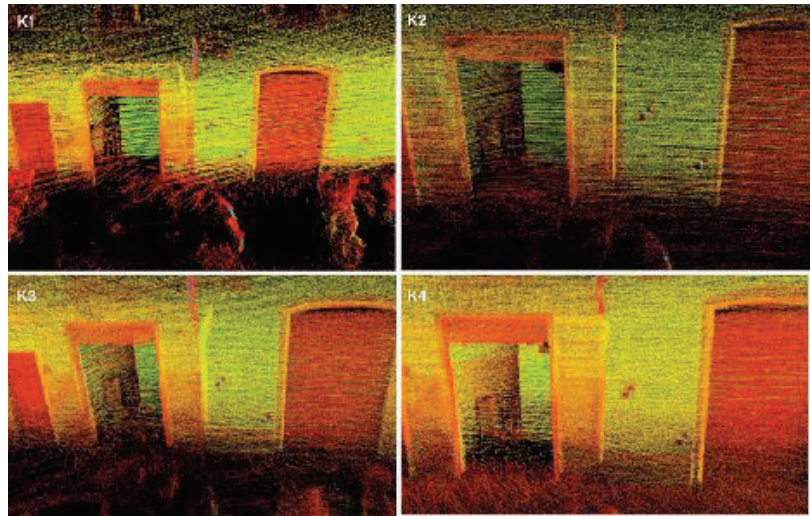

Figure 6. It is possible to observe the variation of the density of the point cloud and consequently the readability of the data in relation to the time of and the number of paths taken.

In the first slot, the third level of the front is completely illegible, it is possible to read the openings of the second and first level but without any details. In the second scan slot, you can read the openings present on all levels. Still, it is easy to report a dimensional error in the representation of the third-level windows during the drawing phase due to the poor readability of the data.
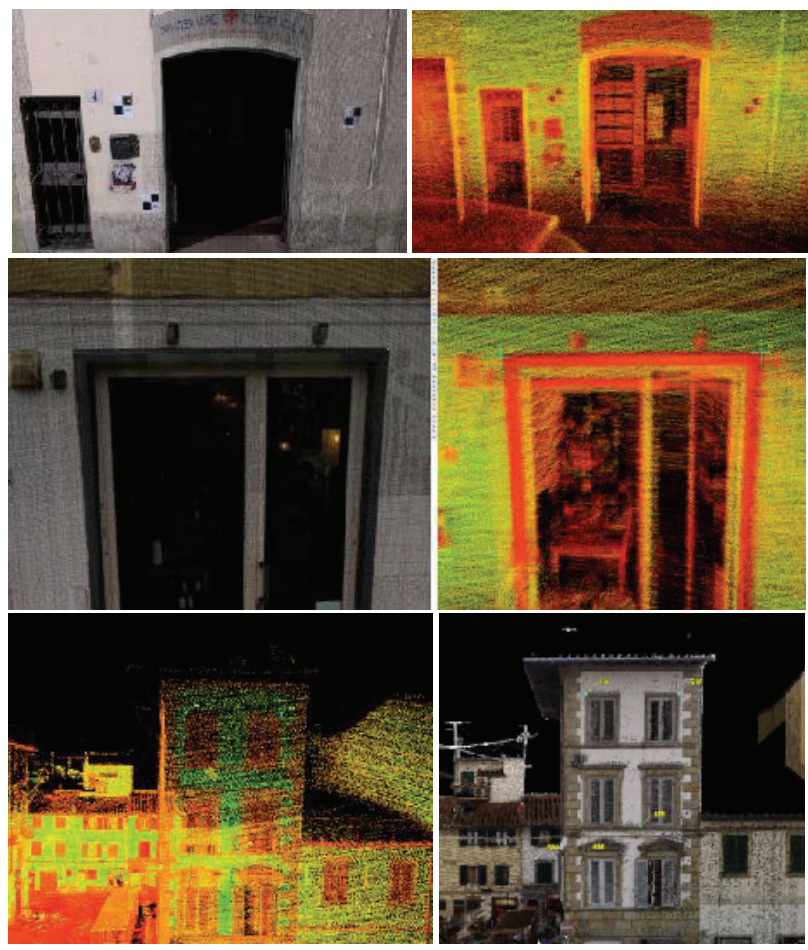

Figure 7. Comparison of how the images appear extrapolated from the point cloud generated by the mobile laser scanner

(KAARTA Stencil) and from the RGB acquisition of the terrestrial laser scanner (FARO Cam Focus S150).

From the third to the fourth slot, in which the density of points is tripled compared to the previous ones, the representation becomes more reliable and legible even with details such as the 

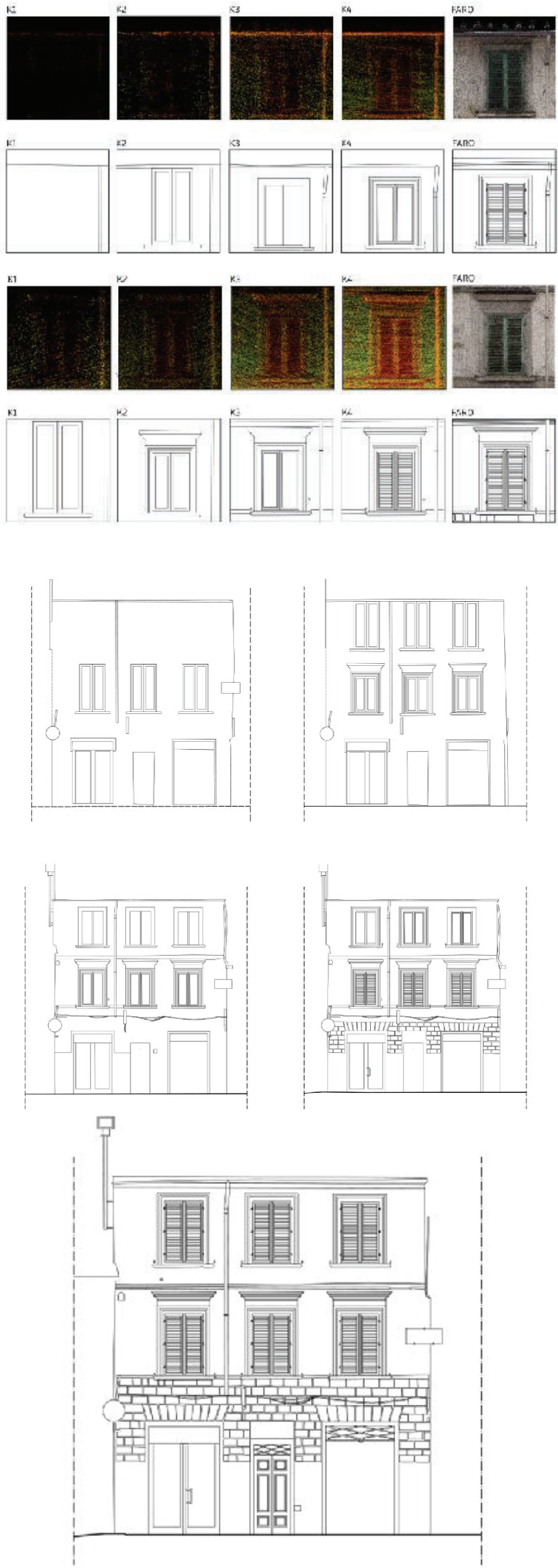

Figure 8. Compartmental diagram of the reading and the different possibilities of representation based on the different types of acquisitions made. Two windows were chosen on the

front at the third and second level to evaluate the data. acquisition range; the same was then done for the entire portion of the urban front. presence of fixtures and cornices. In the fourth slot, it is possible to elaborate a more detailed review by adding the stone cladding of the base and some details such as the handle of the entrance door to the shop.

\section{CONCLUSION AND FINAL RESULTS}

This result encourages the use of mobile systems for urban representation. In particular, they allow rapid acquisition actions for the documentation of the historical and cultural heritage at an urban scale, allowing to relate to different urban contexts, overcoming the difficulties related to reduced accessibility or an amplitude such that it cannot be covered in an adequate time by the $3 \mathrm{D}$ survey procedures consolidated over time through the use of terrestrial laser scanners (TLS) (di Filippo et al 2018).

The accuracy of the data has been evaluated at two different levels: between different slot of mobile scan, and between the mobile data in comparison with the TLS laser scanner, have been confronted. Future investigations could concern the use of the point clouds for the automatic detection of surface for the development of the point cloud data into building information modelling. Furthermore, the advent of information modeling systems has encouraged the development of technologies aimed at fast 3D digitization, which encourages the design of tools for data acquisition that aim to reduce acquisition and time while maintaining a good quality of the detail level of the morphological surface. Mobile devices are born for the approach defined Scan-to-BIM, according to which the geometric data is derived directly from the point cloud or through automatic recognition processes through specific software or the extrusion and modeling of local components inside of the model project, without resorting to pre-established object libraries (Osello et al., 2017).

On the other hand, the mobile tool aligns itself with the process of renewal of the information message of digital representation models, addressing again those tensions that characterize their formal identity, modifying the metric morphological reliability and the qualities that increase its verisimilitude and correspondence to the real, as a function of more characteristic parameters of other interpretative aspects not strictly connected to the form. If, on the one hand the tendency is to make the digital model correspond as closely as possible to the real object, the synthesis of complexity is oriented on criteria of approximation of the form and limitation of imperfection to the advantage of standardization of the formal components of the built and easier computation and interaction with the model element (Miceli et al. 2020).

These are the communication criteria that define the methods of construction of the form to find in the graphics and, therefore, the definition of a graphic language of the helpful model words to make concepts that can also be complex easier to understand. The digital representation model faces again those tensions that characterize its formal identity, modifying the metric morphological reliability and the qualities that increase its verisimilitude and correspondence to reality, according to more characterizing parameters other aspects not strictly connected to the form. If on the one hand the tendency is to make the digital model correspond as closely as possible to the real object, the synthesis of complexity is oriented on criteria of approximation of the form and limitation of imperfection to the advantage of standardization of the formal components of the built and easier computation and interaction with the model element. (Dell'Amico et al. 2021) 


\section{REFERENCES}

Apollonio, F., Gaiani, M., Remondino, F., 2010. Una pipeline per l'acquisizione di dati in 3D. In: Benedetti, B., Gaiani, M, Remondino, F., (eds) Modelli digitali $3 D$ in archeologia: il caso di Pompei. Edizioni della Normale, Pisa, pp. 39-63.

Sammartano, G., Spanò, A. 2018. Point clouds by SLAM-based mobile mapping systems: accuracy and geometric content validation in multisensor survey and stand-alone acquisition. Appl Geomat 10, pp. 317-339. https://doi.org/10.1007/ s12518-018-0221-7

De Luca, L., Lo Buglio, D., 2014. Geometry vs Semantics: Open Issues on 3D Reconstruction of Architectural Elements. 10.1007/978-3-662-44630-0_3.

Dell'Amico, A., 2020. The application of fast survey technologies for urban surveying: the documentation of the historic center of Santa Cruz de Mompox. In: Barba S., Parrinello S., Limongiello M., Dell'Amico, A., (eds) D-SITE Drones - Systems of Information on culTural hEritage. For a spatial and social investigation. Pavia University Press, Pavia., pp. 132-141.

di Filippo, A.; Sánchez-Aparicio, L.; Barba, S.; Martín-Jiménez, J.; Mora, R.; González Aguilera, D. 2018. Use of a Wearable Mobile Laser System in Seamless Indoor 3D Mapping of a Complex Historical Site. Remote Sens. 2018, 10, 1897

López, J., Barrera-Vera, J. ,(2020). Evaluación de los Sistemas de Mapeo Móvil (MMS) en la documentación gráfica del tholo de El Romeral (Conjunto Arqueológico Dólmenes de Antequera). In: Arqueología de la Arquitectura. 095. 10.3989/ arq.arqt.2020.004.

Lo Turco, M., Calvano, M., Giovannini, C., Tomalini, A., 2021. AIM! Algorithmic Information Modeling: New Strategies for a Fully Integrated Approach in the Field of Cultural Heritage. In: Bolognesi C., Villa A. (eds) From Building Information Modelling to Mixed Reality. Cham, Springer.

Miceli, A., Morandotti, M., Parrinello, S., 2020. 3D survey and semantic analysis for the documentation of built heritage. The case study of Palazzo Centrale of Pavia University. VITRUVIO - International Journal of Architectural Technology and Sustainability, [S.1.], v. 5, n. 1, p. 65-80, june 2020. ISSN 2444-9091.

Osello, A., Ugliotti, F. M, 2017. BIM: verso il catasto del futuro. Conoscere, digitalizzare, condividere. Il caso studio della Città di Torino. Roma, Gangemi Editore.

Parrinello, S., La Placa, S., 2020. Ricostruire la memoria dello Stato da Mar attraverso un percorso di conoscenza, documentazione e disegno/Rebuilding the Memory of the State da Mar through a path of knowledge, documentation and drawing. In: Arena A., Arena M., Brandolino R.G., Colistra D., Ginex G., Mediati D., Nucifora S., Raffa P., (eds). Connettere. Un disegno per annodare e tessere. Atti del $42^{\circ}$ Convegno Internazionale dei Docenti delle Discipline della Rappresentazione/Connecting. Drawing for weaving relationships. Proceedings of the 42th International Conference of Representation Disciplines Teachers. Milano, FrancoAngeli, pp. 2533-2550.

Parrinello S., Dell'Amico, A., 2021. From Survey to Parametric Models: HBIM Systems for Enrichment of Cultural Heritage Management. In: Bolognesi C., Villa A. (eds) From
Building Information Modelling to Mixed Reality. Cham, Springer.

Parrinello, S., Picchio, F., 2019. INTEGRATION AND C O M PAR I S ON O F C L O S E-R A N G E S F M METHODOLOGIES FOR THE ANALYSIS AND THE DEVELOPMENT OF THE HISTORICAL CITY CENTER OF BETHLEHEM. ISPRS - International Archives of the Photogrammetry, Remote Sensing and Spatial Information Sciences. XLII-2/W9. 589-595. 10.5194/isprs-archives-XLII-2W9-589-2019.

Toschi, I., Ramos, M. M., Nocerino, E., Menna, F., Remondino, F., Moe, K., Poli, D., Legat, K., and Fassi, F., 2017. Oblique photogrammetry supporting $3 \mathrm{D}$ urban reconstruction of complex scenarios. In: Int. Arch. Photogramm. Remote Sens. Spatial Inf. Sci., XLII-1/W1, 519-526, https://doi.org/10.5194/ isprs-archives-XLII-1-W1-519-2017, 2017.

Tu, C., Takeuchi, E., Miyajima, E., Takeda, K., 2017. Continuous point cloud data compression using SLAM based prediction IEEE Intelligent Vehicles Symposium (IV), Los Angeles, CA, pp. 1744-1751, doi: 10.1109/IVS.2017.7995959.

Tucci, G., Bonora, V., Conti, A., Fiorini, L., 2017. Digital workflow for the acquisition and elaboration of $3 \mathrm{D}$ data in a monumental complex: the fortress of Saint John the Baptist in Florence. In: ISPRS - International Archives of the Photogrammetry, Remote Sensing and Spatial Information Sciences, XLII-2/W5, 679-686. https:// doi.org/10.5194/isprsarchives-XLII-2-W5-679-2017. 